\title{
Effect of bisacodyl on rats with slow transit constipation
}

\author{
Yong-bing Wang ${ }^{1}$, Jie Ling ${ }^{1}$, Wen-zhong Zhang ${ }^{1}$, Gang $\mathrm{Li}^{1}$, Wei Qiu ${ }^{1}$, Jun-hua Zheng ${ }^{1}$ and \\ Xiao-hui Zhao ${ }^{2}$ \\ ${ }^{1}$ Department of General Surgery, Pudong New Area People's Hospital, Shanghai University of Medicine \& Health Sciences, \\ Shanghai, China \\ ${ }^{2}$ Clinical Medical School, Shanghai University of Medicine \& Health Sciences, Shanghai, China
}

\begin{abstract}
The effect of bisacodyl on the treatment of rats with slow transit constipation (STC) was studied. Forty-five female Wister rats were divided into control group, STC group, and STC bisacodyl group. The immunohistochemical method was used to determine interstitial cells of Cajal (ICC) and the expression of c-Kit protein. Body mass and the number of defecations were significantly decreased in the STC group compared with the control group on the 100th day after diphenoxylate administration, while dry weight of feces was significantly increased and the intestinal transit time was prolonged. There were significant differences in the number of defecations, dry weight of feces, and intestinal transit time among the three groups. The number of defecations was higher, dry weight of feces was lower, and intestinal transit time was shorter in the STC bisacodyl group compared to the STC group. In addition, ICC basement membrane dissolution occurred in the colon wall of the STC group. The connection between ICC and surrounding cells was destroyed, and the nucleus shrunken to different degrees. Moreover, c-Kit expression in the STC group was significantly lower than the control group. The connection between ICC and surrounding cells in the STC bisacodyl group was significantly stronger than the STC group, and the number of ICC and the expression of c-Kit were increased. Bisacodyl could reduce the severity of STC in rats by increasing the number of ICC and the expression of c-Kit.
\end{abstract}

Key words: Bisacodyl; Slow transit constipation; c-Kit

\section{Introduction}

Slow transit constipation (STC) is an intractable condition characterized by prolonged transit time of colonic contents that is caused by the decrease of colonic motility. STC can induce abdominal distention, stomachache, defecation difficulty, perianal diseases, and is related with cardiovascular and cerebrovascular diseases and colon cancer (1). The etiology of STC is complex and its mechanism is still not fully understood. Many studies showed that the etiology of STC is related with enteric nervous system, hormones and neurotransmitters, intestine smooth muscle, and interstitial cells of Cajal (ICC) $(2,3)$. The ICC are pacemaker cells that produce gastrointestinal myoelectric activity and gastrointestinal motility, contributing to colon motility and fecal propulsion. Researchers have found that ICC could participate in STC. For example, Zhu et al. (4) reported that total glucosides of paeony promoted intestinal motility in STC rats through amelioration of ICC and Geramizadeh et al. found that ICC was decreased in patients with STC (5). Therefore, we focused on ICC to reveal the underlying mechanism of STC.

Bisacodyl is a common drug for the treatment of STC, which was first used in 1985. Later, researchers found that bisacodyl promoted and propagated motor activity in patients with severe STC (6). However, the mechanism of bisacodyl on ICC is not revealed and there is still a lack of adequate clinical data.

Rats have a mild disposition, good tolerance, and high success rate in experimental research, therefore they are the first choice for the study of STC (7). The STC rat model was established by continuously feeding diphenoxylate, which can be applied in clinical practice and has many advantages, such as being a simple, economical, and reproducible method (4).

In this study, we established an STC rat model and treated STC rats with bisacodyl. Our aim was to study the relationship between bisacodyl and ICC, and explore the potential underlying mechanism of STC.

\section{Material and Methods}

\section{Animals}

Female Wister rats, weighing 120-140 g, were purchased from Shanghai Laboratory Animal Center of Chinese Academy of Science (China), and kept in 50-70\% humidity at room temperature. All procedures were approved by 
the Ethics Committee of the Pudong New Area People's Hospital.

\section{Establishment of the STC rat model}

Forty-five female Wister rats were kept in separate cages for three days. Thirty rats were divided into two groups: STC group and STC bisacodyl group. The other 15 rats were used as the control group.

The rats in the STC groups were given diphenoxylate by intragastric administration $\left(8 \mathrm{mg} \cdot \mathrm{kg}^{-1} \cdot \mathrm{d}^{-1}\right)$, while rats in the control group were treated with the same amount of physiological saline orally. After 100 days of diphenoxylate treatment, rats in the STC bisacodyl group were treated with bisacodyl $\left(20 \mathrm{mg} \cdot \mathrm{kg}^{-1} \cdot \mathrm{d}^{-1}\right)$ for 30 days. The number of defecations, feces dry weight, and body weight of rats were recorded every day. One week after drug withdrawal, the rats were fasted for $24 \mathrm{~h}$. The rats received $100 \mathrm{~g} / \mathrm{L}$ activated carbon $(2 \mathrm{~mL})$ and then the time from the intragastric administration to the first black stool was recorded. The rats were caged individually and the number of defecations was measured by the metabolic cage (pellet/ day). Feces were dried by natural air at room temperature and then weighed (mg/pellet).

\section{Measurement of C-Kit positive ICC and C-Kit expression in the colon}

After one month of bisacodyl treatment, rats were anesthetized with $2 \%$ pentobarbital via intraperitoneal injection. The abdominal cavity was exposed through an abdominal median incision. Intestinal tissue (about $1 \mathrm{~cm}$ length) was cut from the colon $5 \mathrm{~cm}$ away from ileocecal valve. Then, the intestinal tissue was washed with PBS and fixed with $4 \%$ paraformaldehyde for paraffin embedding.

The sections (about $5 \mu \mathrm{m}$ ) were mounted on slides and baked on a $75^{\circ} \mathrm{C}$ baking instrument (Boxin Equipment Inc., China) for $10 \mathrm{~min}$. Xylene solution was used for dewaxing. The procedures for hematoxylin-eosin staining were as follows: hydration for $10 \mathrm{~s}$ with anhydrous ethanol, $95 \%$ ethanol, and $80 \%$ ethanol; wash with water for 2 min; hematoxylin staining for $8 \mathrm{~min}$; wash with flowing water for $1 \mathrm{~min} ; 1 \%$ hydrochloric acid ethanol for $5 \mathrm{~s}$; wash with flowing water for $20 \mathrm{~s}$; $1 \%$ eosin staining for $2 \mathrm{~min}$; wash with water for $2 \mathrm{~min}$; dehydration of gradient ethanol for $3 \mathrm{~min}$; dehydration of xylene for $5 \mathrm{~min}$; and seal with neutral gum.

The procedures of c-Kit protein immunohistochemical staining were as follows: dewax and dewater the sections; soak with PBS for 5 min; sodium citrate for restoration; natural cooling for $5 \mathrm{~min}$; wash with cold water for $10 \mathrm{~min}$; soak with PBS for $5 \mathrm{~min}$; incubate with $3 \%$ hydrogen peroxide solution for $15 \mathrm{~min}$; wash with PBS for $2 \mathrm{~min}$; add rabbit anti-mouse c-Kit polyclonal antibody (Thermo Scientific, USA) and incubate for $1.5 \mathrm{~h}$; wash with PBS for $2 \mathrm{~min}$; add enzyme labeled second antibody for $30 \mathrm{~min}$; wash with PBS for 2 min; add diaminobenzidine (DAB) colored solution for $10 \mathrm{~min}$; wash with distilled water; hematoxylin for counterstaining for $5 \mathrm{~min}$; dehydration of gradient ethanol for $5 \mathrm{~min}$; dehydration of xylene for $5 \mathrm{~min}$; wash with PBS for 3 times; and seal with neutral gum.

Specimens were observed under a light microscope $(\times 100)$. Blue nucleus and brown cytoplasm were determined as c-Kit-positive cells (ICC cells). Five high magnification views $(\times 100)$ were randomly selected in each slice, and the number of ICC cells was calculated in each field. c-Kit expression was proportional to the area or concentration of the brown cytoplasm. The areas of five high magnification fields $(\times 100)$ were measured by ImagePro Plus 6.0, and the change of c-Kit expression is reported as the percentage of area size.

\section{Statistical analysis}

SPSS, version 20.0, was used for data analysis (USA). Measurement data are reported as means \pm SD. For normally distributed data, differences between two groups were tested using Student's $t$-test, and differences among three groups were tested by one-way ANOVA. Nonnormally distributed data were analyzed by non-parametric analyses, with $\mathrm{P}<0.05$ considered statistically significant.

\section{Results}

\section{Body mass of STC rats}

As shown in Figure 1 , at the $50^{\text {th }}$ and $100^{\text {th }}$ day after feeding diphenoxylate suspension, body mass in STC rats was significantly lower than control rats $(321.9 \pm 13.3 \mathrm{~g}$ vs $337.6 \pm 12.7 \mathrm{~g} ; \mathrm{P}=0.013$ and $389.6 \pm 15.1 \mathrm{~g}$ vs 482.9 $\pm 11.5 \mathrm{~g}$, respectively; $P=0.000$ ). These findings indicated that the STC model was successfully established.

\section{Defecation condition}

At the $100^{\text {th }}$ day after feeding diphenoxylate suspension, the number of defecations per day in the STC group was less than that of the control group $(P<0.05)$, while dry



Figure 1. Changes of body mass in slow transit constipation (STC) rats and control rats after feeding diphenoxylate suspension. Data are reported as means $\pm S D$. ${ }^{*} P<0.05$ compared to control (ANOVA). 
weight of feces and intestinal transit time were greater than that of the control group $(P<0.05)$ (Table 1).

After one month of bisacodyl treatment, there were significant differences in the number of defecations, dry weight of feces, and intestinal transit time among the control group, STC group, and STC bisacodyl group $(P=0.000)$. The number of defecations in the STC bisacodyl group was greater than that of the STC group $(P<0.05)$, while dry weight of feces and intestinal transit time in the STC bisacodyl group were less than in the STC group $(P<0.05)$ (Table 2).

\section{Comparison of pathological characteristics and C-Kit expression}

In the STC group, the ICC basement membrane was dissolved in the colon wall. The connection between ICC and surrounding cells was destroyed, and the nucleus was shrunken to different degrees. In the STC group, the number of ICC in a single field of vision was $9.51 \pm 2.08$, and c-Kit expression was $(13.05 \pm 3.12 \%)$, which were significantly lower than the control group $(37.12 \pm 5.36$, $72.01 \pm 9.33 \%)(P=0.000)$.

Compared with the STC group, the connection between ICC and surrounding cells in the STC bisacodyl group was significantly strengthened, and the number of ICCs (19.04 $\pm 3.51)$ and the expression of c-Kit $(46.28 \pm 7.09 \%)$ were also increased $(\mathrm{P}=0.000)$ (Figure 2).

\section{Discussion}

Constipation can severely reduce life quality of the patients and bring financial burden to the patients and their families $(8,9)$. Recently, attention has been paid to the influence of STC on the health of patients, and further investigations are still needed to understand the pathogenesis and find an effective treatment $(10,11)$. Our previous report showed that intestinal transit time of STC patients was significantly longer than healthy volunteers, while the frequency, contraction amplitude, and physiological responsivity of high amplitude propagated constriction (HAPC) were less than in healthy volunteers, which suggested that dynamic abnormality was a major cause of slow colonic transit and could increase constipation in STC patients (12).

Bisacodyl is a new drug that increases intestinal motility by the following possible mechanisms (13): increasing prostaglandin $\mathrm{E} 2$, thereby reducing colonic aquaporin 3 and promoting colonic motility, and decreasing the absorption of sodium ions, chloride ions, and moisture in colon by increasing intestinal volume. Bisacodyl or its product decomposed by intestinal bacteria can directly stimulate the intestinal wall innervation, mediate the reflex, cause intestinal reflex peristalsis, and result in defecation (14). Bisacodyl could change the migrating motor complex of colonic mobility by increase of HAPC (15). Therefore, it

Table 1. Comparison of defecation condition in control and slow transit constipation (STC) rats after 100 days of diphenoxylate treatment.

\begin{tabular}{lccc}
\hline Groups & $\begin{array}{c}\text { Number of defecations } \\
\text { (pellets/d) }\end{array}$ & $\begin{array}{c}\text { Dry weight of feces } \\
(\mathrm{mg} / \text { pellet })\end{array}$ & $\begin{array}{c}\text { Intestinal transit time } \\
(\mathrm{min})\end{array}$ \\
\hline Control $(\mathrm{n}=15)$ & $41.4 \pm 5.4$ & $145.8 \pm 21.7$ & $408.8 \pm 64.9$ \\
STC $(\mathrm{n}=30)$ & $24.8 \pm 5.7$ & $193.1 \pm 15.8$ & $515.5 \pm 100.2$ \\
$t$ value & 6.667 & 5.576 & 2.826 \\
$\mathrm{P}$ value & 0.000 & 0.000 & 0.011 \\
\hline
\end{tabular}

Data are reported as means $\pm \mathrm{SD}$. Between-group comparison was done with the $t$-test.

Table 2. Comparison of defecation condition among control, slow transit constipation (STC) control, and STC bisacodyl groups after 1 month of diphenoxylate treatment.

\begin{tabular}{lccc}
\hline Groups & $\begin{array}{c}\text { Number of } \\
\text { defecations }(\text { pellets } / \mathrm{d})\end{array}$ & $\begin{array}{c}\text { Dry weight of feces } \\
(\mathrm{mg} / \mathrm{pellet})\end{array}$ & $\begin{array}{c}\text { Intestinal transit time } \\
(\mathrm{min})\end{array}$ \\
\hline Control $(\mathrm{n}=15)$ & $45.3 \pm 8.0$ & $126.6 \pm 17.7$ & $358.2 \pm 47.7$ \\
STC $(\mathrm{n}=15)$ & $25.9 \pm 5.8$ & $172.3 \pm 15.8$ & $496.8 \pm 65.1$ \\
STC bisacodyl $(\mathrm{n}=15)$ & $37.2 \pm 5.4^{*}$ & $151.1 \pm 9.3^{*}$ & $415.4 \pm 48.2^{*}$ \\
$t$ value & 17.60 & 21.94 & 15.27 \\
P value & 0.000 & 0.000 & 0.000 \\
\hline
\end{tabular}

Data are reported as means $\pm S D$. ${ }^{*} \mathrm{P}<0.05$, compared with STC group (ANOVA). 

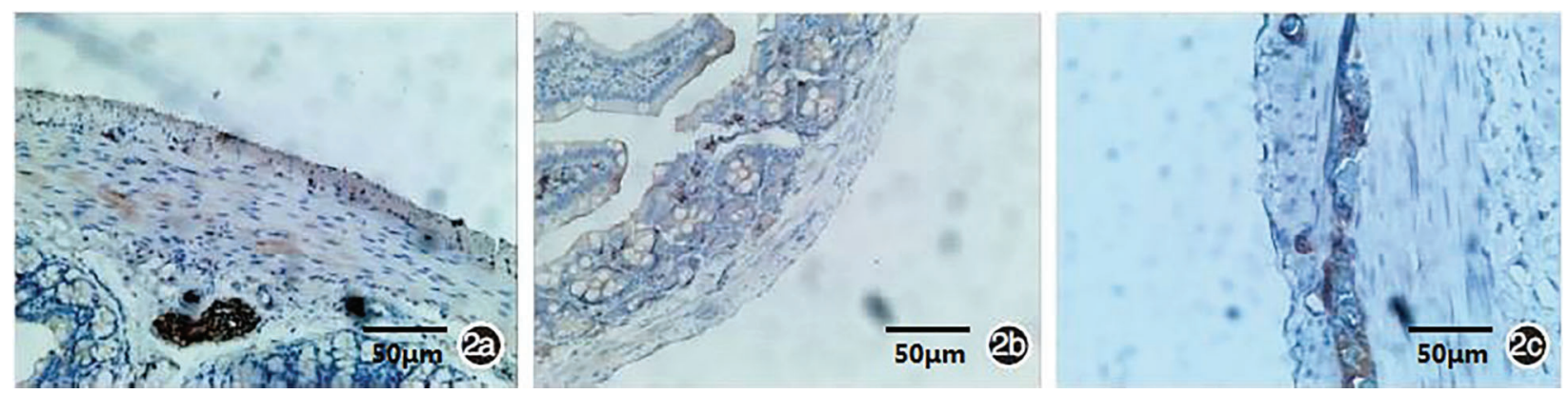

Figure 2. Immunohistochemical images of c-Kit protein in rat colon cells $(\times 200$, bar: $50 \mu \mathrm{m})$. $2 a$, Slow transit constipation (STC) bisacodyl group; $2 b$, STC group; $2 c$, Control group.

was speculated that long-term use of bisacodyl improves the colonic motility and ICC function.

In the current study, the 100th day, when STC rats had the most pronounced difference in body mass, was selected as the experimental time point to observe the effect of bisacodyl. The symptoms of STC rats were significantly relieved at the 30th day after feeding bisacodyl, because the number of defecations increased, dry weight of feces decreased and the intestinal transit time shortened. These findings indicated that bisacodyl improved the constipation of rats and had a good therapeutic effect.

The digestive tract, like the heart, has natural pacemakers (16). The contractile activity of the digestive tract is controlled by the slow wave of the pacemakers (17). The action potential superimposed on the slow wave potential induces contraction of smooth muscles of the digestive tract. Early in 1982, Thuneberg pointed out that ICC are the pacemaker that produce gastrointestinal myoelectric activity, which was proven by the electrophysiological test in vitro and in vivo (18). Reduction and dysfunction of ICC could result in a decrease in slow wave activity in the colon, and block information transmission between intestinal nervous system and smooth muscle cells, delaying electrical excitement transmission between pacemaker cells and smooth muscle cells, and resulting

\section{References}

1. Stern T, Davis AM. Evaluation and Treatment of Patients With Constipation. Jama 2016; 315: 192-193, doi: 10.1001/ jama.2015.16995.

2. Costilla VC, Foxx-Orenstein AE. Constipation: understanding mechanisms and management. Clin Geriatr Med 2014, 30: 107, doi: 10.1016/j.cger.2013.10.001.

3. Bassotti G, Blandizzi C. Understanding and treating refractory constipation. World J Gastrointest Pharmacol Ther 2014; 5: 77-85, doi: 10.4292/wjgpt.v5.i2.77.

4. Zhu F, Xu S, Zhang Y, Chen F, Ji J, Xie G. Total glucosides of paeony promote intestinal motility in slow transit constipation rats through amelioration of interstitial cells of cajal. in decreased contractility of smooth muscle cells, colonic motility disorder, and fecal transit delay.

The c-Kit receptor is a transmembrane protein encoded by proto-oncogene c-kit that has tyrosine kinase activity (19). It is a marker of ICC, and can be detected by a specific antibody (20). The number of ICC in STC rats was decreased, and c-Kit expression was decreased, consistent with a previous report (5). These findings suggested that STC was related with the number of ICC and C-Kit expression. In the current study, the number of ICC and c-Kit expressions in the STC bisacodyl group were significantly increased, which indicated that the effect of bisacodyl on the treatment of STC was closely related to the number of ICC and C-Kit expressions, and provided theoretical basis for bisacodyl treatment of STC.

As a limitation, we did not observe the time for reversal of the STC model after stopping diphenoxylate treatment and there is a possibility that part of the response of bisacodyl is due to this time.

\section{Acknowledgments}

This study was supported by Key Discipline Construction Program of Pudong Health Bureau of Shanghai (No. PWZxk2017-11) and Medical Key Specialty of Shanghai (No. 2015-B-18).
PloS One 2016, 11: e0160398, doi: 10.1371/journal.pone. 0160398.

5. Geramizadeh B, Hayati K, Rahsaz M, Hosseini SV. Assessing the interstitial cells of Cajal, cells of enteric nervous system and neurotransmitters in slow transit constipation, using immunohistochemistry for CD117, PGP9.5 and serotonin. Hepatogastroenterology 2009; 56: 16701674.

6. Herve S, Savoye G, Behbahani A, Leroi AM, Denis P, Ducrotte $P$. Results of 24-h manometric recording of colonic motor activity with endoluminal instillation of bisacodyl in patients with severe chronic slow transit constipation. 
Neurogastroenterol Motil 2004; 16: 397-402, doi: 10.1111/ j.1365-2982.2004.00535.x.

7. Zhang YG, Shao WJ, Gu YF, Qiu JF, Yuan L, Li GD. Effects of sacral nerve stimulation with acupuncture on gut transit time and c-kit expression in colon of rats with slow transit constipation. Genet Mol Res 2016; 15.

8. Baffy N, Foxx-Orenstein AE, Harris LA, Sterler S. Intractable constipation in the elderly. Curr Treat Options Gastroenterol 2017; 15: 363-381, doi: 10.1007/s11938-017-0142-2.

9. Lu PL, Koppen IJN, Orsagh-Yentis DK, Leonhart K, Ambeba EJ, Deans KJ, et al. Sacral nerve stimulation for constipation and fecal incontinence in children: Long-term outcomes, patient benefit, and parent satisfaction. Neurogastroenterol Motil 2018; 30, doi: 10.1111/nmo.13184.

10. Patton V, Stewart P, Lubowski DZ, Cook IJ, Dinning PG. Sacral nerve stimulation fails to offer long-term benefit in patients with slow-transit constipation. Dis Colon Rectum 2016; 59: 878-885, doi: 10.1097/DCR.000000000000 0653.

11. Hutson JM, Dughetti L, Stathopoulos L, Southwell BR. Transabdominal electrical stimulation (TES) for the treatment of slow-transit constipation (STC). Pediatr Surg Int 2015; 31: 445-451, doi: 10.1007/s00383-015-3681-4.

12. Wang $Y$, Zhang G, Jiang $P$, Yan G, Zhu Z, Zhang M, et al. Applications of a gastrointestinal monitoring microsystem based on the smart capsule in measuring the pressure of colon and the transmission function of colon. Chin J Gen Surg 2008; 28: 171-206.

13. Soufi-Afshar I, Moghadamnia A, Bijani A, Kazemi S, ShokriShirvani J. Comparison of pyridostigmine and bisacodyl in the treatment of refractory chronic constipation. Caspian $J$ Intern Med 2016; 7:19-24.

14. Ikarashi N, Baba K, Ushiki T, Kon R, Mimura A, Toda T, et al. The laxative effect of bisacodyl is attributable to decreased aquaporin-3 expression in the colon induced by increased PGE2 secretion from macrophages. Am J Physiol Gastrointest Liver Physiol 2011; 30: G887-895, doi: 10.1152/ajpgi.00286.2011.

15. Bharucha AE. High amplitude propagated contractions. Neurogastroenterol Motil 2012; 24: 977, doi: 10.1111/nmo. 12019.

16. Mane N, Jimenez M. Interplay between myogenic pacemakers and enteric neurons determine distinct motor patterns in the rat colon. Neurogastroenterol Motil 2014; 26: 15081512, doi: $10.1111 / \mathrm{nmo} .12393$.

17. Angeli TR, O'Grady G, Du P, Paskaranandavadivel N, Pullan AJ, Bissett IP, et al. Circumferential and functional re-entry of in vivo slow-wave activity in the porcine small intestine. Neurogastroenterol Motil 2013, 25: e304-314, doi: $10.1111 / \mathrm{nmo} .12085$.

18. Thuneberg L. Interstitial cells of Cajal: intestinal pacemaker cells? Adv Anat Embryol Cell Biol 1982; 71: 1-130, doi: 10.1007/978-3-642-68417-3.

19. Liang J, Wu YL, Chen BJ, Zhang W, Tanaka Y, Sugiyama H. The c-kit receptor-mediated signal transduction and tumorrelated diseases. Int J Biol Sci 2013; 9: 435-443, doi: 10.7150/ ijbs.6087.

20. Radenkovic G, Savic V, Mitic D, Grahovac S, Bjelakovic M, Krstic M. Development of c-kit immunopositive interstitial cells of Cajal in the human stomach. J Cel Mol Med 2010; 14: $1125-1134$. 